\title{
Dinâmica trófica de Plagioscion squamosissimus (Perciformes, Sciaenidae) em trechos de influência da represa Capivara (rios Paranapanema e Tibagi)
}

\author{
Sirlei T. Bennemann, Luiz G. Capra, Wanner Galves \& Oscar A. Shibatta
}

Programa de Pós-Graduação em Ciências Biológicas, Departamento de Biologia Animal e Vegetal, Centro de Ciências Biológicas, Universidade Estadual de Londrina, Rod. Celso Garcia Cid, 86.051-990 Londrina, PR, Brasil. (sirlei@uel.br)

\begin{abstract}
Trophic dynamic of Plagioscion squamosissimus (Perciformes, Scianidae) in stretches under influence of the Capivara dam (Paranapanema and Tibagi rivers). Plagioscion squamosissimus (Heckel, 1840) was studied in five stretches in Paranapanema and Tibagi rivers under the influence of the Capivara reservoir. The samplings were made on a monthly basis during the $1992 / 1993$ and 1994/1995 periods and seasonally during the 2001/2002 period. The captures were made with gillnets with meshes varying between 2 and $12 \mathrm{~cm}$. A total of 993 specimens had their stomach analyzed. The identified food items were grouped in trophic categories and analyzed by the percent composition method. The diet composition was compared among sampling periods and among stretches by the similarity analysis using the Bray-Curtis' coefficient. The results indicated that the consumption of food items was uniform in all periods and stretches analysed. The food items found were grouped in six categories: fishes, shrimp, Odonata, Ephemeroptera, other insect groups and "others" (plant material, detritus and rarely-found organisms). In all stretches and during all the studied periods, P. squamosissimus presented a carnivorous diet, and during the 2001/2002 period the item shrimp (Macrobrachium amazonicum) represented the largest portion of the diet. Concomitant with the shift from fish to shrimp, there were also changes in feeding behavior, shown by the changes in feeding frequency in food taking and in the variety of consumed food items. A large number of prey individuals were found in the stomach contents when P. squamosissimus fed on shrimp, with most specimens feeding exclusively on this food item.
\end{abstract}

KEYWORDS. Introduced species, pescada-do-Piauí, non-native fish, freshwater, predation.

RESUMO. Plagioscion squamosissimus (Heckel, 1840) foi estudada em cinco trechos de influência da represa Capivara nos rios Paranapanema e Tibagi. As amostragens foram realizadas em dois períodos, 1992/1993 e 1994/1995, em coletas mensais, e sazonalmente no período 2001/2002. As capturas foram efetivadas com redes de espera simples com malhas entre 2 a $12 \mathrm{~cm}$. Foram analisados 993 exemplares. Os itens alimentares identificados foram agrupados em categorias e analisados pelo método da composição percentual. A composição da dieta foi comparada entre os períodos de amostragens e entre trechos, pela análise de similaridade usando o coeficiente de Bray-Curtis. Os resultados indicaram que em todos os períodos e em todos os trechos houve uniformidade no consumo dos tipos de itens alimentares. Os itens encontrados foram agrupados em seis categorias: peixes, camarão, Odonata, Ephemeroptera, outros grupos de insetos e outros (material vegetal, detritos e organismos, raramente encontrados). Em todos os trechos e em todas as épocas analisadas, P. squamosissimus se manteve como carnívora, e no período 2001/2002 o item camarão (Macrobrachium amazonicum) foi o de maior participação em sua dieta. Concomitante com o fato de a corvina trocar de alimento substituindo peixes por camarão, mudou o comportamento quanto à freqüência na tomada de alimento e na variedade de tipos de itens consumidos. Quando P. squamosissimus utiliza camarão, maior número de indivíduos são encontrados nos estômagos, na maioria dos casos com apenas este tipo de item.

PALAVRAS-CHAVE. Espécie introduzida, pescada-do-Piauí, peixe não nativo, água doce, predação.

A corvina Plagioscion squamosissimus (Heckel, 1840) é uma espécie natural da bacia Amazônica, mas foi recentemente introduzida na região média da bacia do rio Paranapanema. Nesta bacia ela foi coletada pela primeira vez no ano de 1992, no trecho Sertanópolis do rio Tibagi, cujas características ambientais são influenciadas pelo reservatório formado no rio Paranapanema pela UHE Escola de Engenharia Mackenzie, conhecido comumente como represa Capivara (Bennemann et al., 2000). Desde então, a espécie ocupa lugar de destaque entre as de maior abundância em número de exemplares, tanto neste trecho do rio Tibagi, quanto em diferentes trechos da represa Capivara.

A dinâmica trófica das espécies de peixes de uma zona litorânea do mesmo trecho do rio Tibagi foi analisada por Bennemann \& Shibatta (2002), durante quatro períodos, e foi constatado que poucas espécies tais como P. squamosissimus persistem naquele trecho quando o nível da água diminui e há mudanças nas características do habitat. De acordo com Lowe-McConnell (1999), as alterações abruptas no habitat afetam, principalmente, a disponibilidade de alimentos, e os peixes que permanecem não podem ser grandes especialistas, pois geralmente necessitam alterar rapidamente de alimento.

Considerando os resultados dos estudos realizados no rio Tibagi, em vários períodos, e em trechos diferentes do rio Paranapanema incluindo o reservatório e áreas de sua influência, foi analisada a dinâmica trófica de $P$. squamosissimus com relação à atividade, estratégia alimentar e consumo do alimento (espacial e temporalmente) na tentativa de compreender o comportamento da espécie na bacia desde a sua introdução.

\section{MATERIAL E MÉTODOS}

A área de estudos se localiza na bacia do rio Paranapanema, entre as coordenadas geográficas $22^{\circ} 30^{\prime}$ 
a $23^{\circ} \mathrm{S}$ e $50^{\circ}$ a $51^{\circ} 30^{\prime} \mathrm{W}$ (Fig. 1) e suas características ambientais estão apresentadas na Tab. I.

Os exemplares analisados foram coletados no trecho Sertanópolis do rio Tibagi com freqüência mensal nos períodos de 1992/1993 e 1994/1995 e sazonal no período de 2001/2002, e mensalmente no trecho Primeiro de Maio do rio Tibagi no período de 1994/1995. Os trechos do rio Paranapanema (Cruzália, Cinzas e Porecatu) foram amostrados sazonalmente no período de 2001/2002. As capturas foram efetivadas com redes de espera simples com malhas entre 2 a $12 \mathrm{~cm}$ entre nós opostos, que ficaram expostas por 24 horas. Exemplares testemunhos estão depositados e permanecem como material de referência na coleção de peixes do Museu de Zoologia da
Universidade Estadual de Londrina (MZUEL).

De cada exemplar, após ser identificado, o trato digestório foi retirado e fixado em formol, para posterior análise dos conteúdos estomacais. Para a análise da dieta foi empregado o método da composição percentual, conforme Hynes (1950). Os percentuais totais da composição da dieta foram comparados entre os períodos de amostragens e entre trechos, pela análise de similaridade, por pares agrupados, segundo o coeficiente de Bray-Curtis com o programa PAST (HAMmER et al., 2003). A atividade alimentar foi analisada considerando os percentuais de exemplares com e sem conteúdo estomacal e as médias do número de itens utilizados por indivíduo.

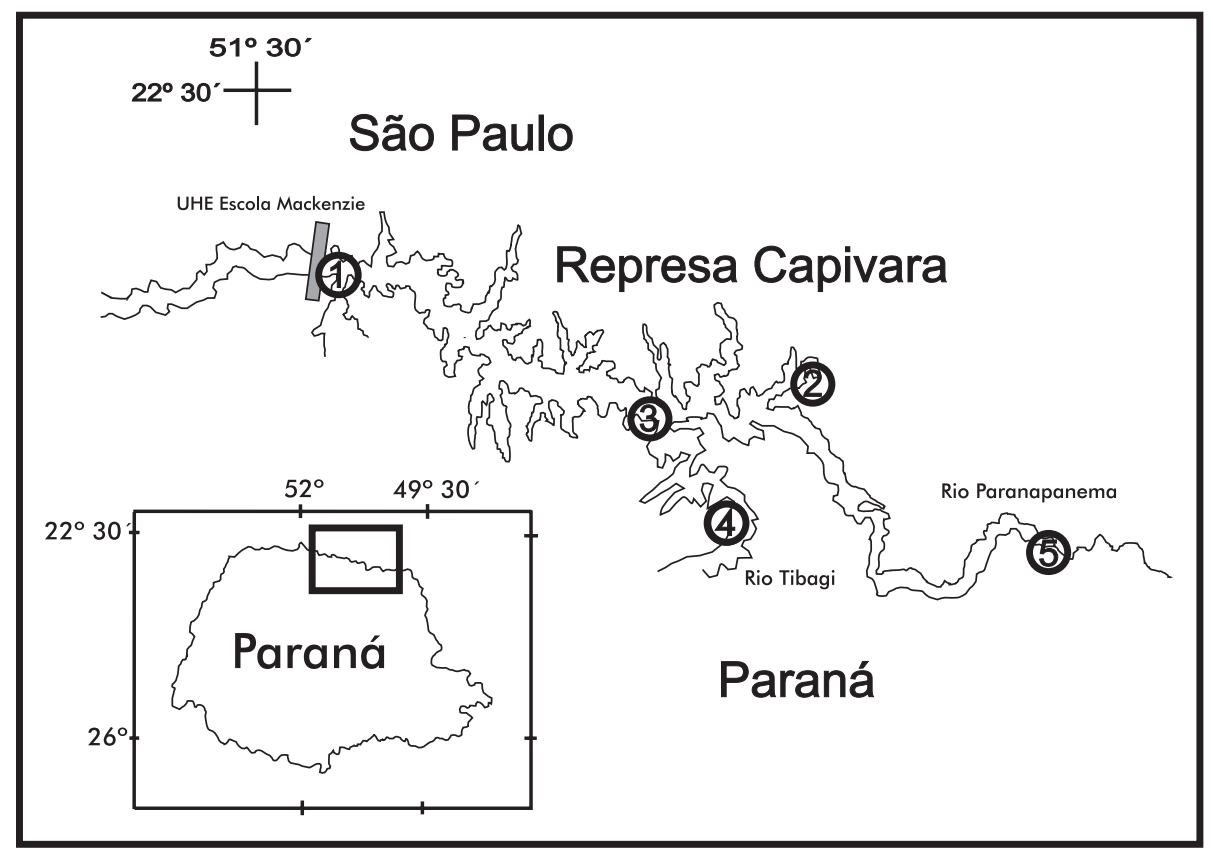

Fig. 1. Detalhe da porção média do rio Paranapanema com a indicação dos trechos de coleta. Porecatu (1); Cruzália (2); Primeiro de Maio (3); Sertanópolis (4) e Cinzas (5).

Tabela I. Características dos trechos amostrados influenciados pela represa Capivara (CIN, Cinzas; CRU, Cruzália; POR, Porecatu ; PM, Primeiro de Maio; SER, Sertanópolis).

\begin{tabular}{|c|c|c|c|c|c|}
\hline \multirow[t]{3}{*}{ Características } & \multicolumn{5}{|c|}{ Trecho amostrado } \\
\hline & \multicolumn{2}{|c|}{ Rio Tibagi } & \multicolumn{3}{|c|}{ Rio Paranapanema } \\
\hline & SER & $\mathrm{PM}$ & CRU & CIN & POR \\
\hline $\begin{array}{l}\text { Profundidade no } \\
\text { local amostrado }\end{array}$ & $\begin{array}{l}\text { Raso até } 4 \mathrm{~m} \text {, com } \\
\text { locais profundos }\end{array}$ & $\begin{array}{l}\text { A maior parte muito } \\
\text { profunda, até } 20 \mathrm{~m}\end{array}$ & Até $8,7 \mathrm{~m}$ & 0,8 a $5,2 \mathrm{~m}$ & $\begin{array}{l}\text { Trechos muito } \\
\text { profundos. Até } 79 \mathrm{~m}\end{array}$ \\
\hline Substrato & $\begin{array}{l}\text { Predominantemente } \\
\text { areno-argiloso }\end{array}$ & $\begin{array}{l}\text { Rochoso e areno- } \\
\text { argiloso }\end{array}$ & $\begin{array}{l}\text { Predominantemente } \\
\text { areno-argiloso }\end{array}$ & $\begin{array}{l}\text { Rochoso e areno- } \\
\text { argiloso }\end{array}$ & $\begin{array}{l}\text { Rochas alternadas } \\
\text { com areia e argila }\end{array}$ \\
\hline Vegetação aquática & $\begin{array}{l}\text { Diversidade e abun- } \\
\text { dância de macrófitas }\end{array}$ & $\begin{array}{l}\text { Poucas macrófitas } \\
\text { aquáticas }\end{array}$ & $\begin{array}{l}\text { Poucas macrófitas } \\
\text { aquáticas }\end{array}$ & $\begin{array}{l}\text { Diversidade e abun- } \\
\text { dância de macrófitas }\end{array}$ & $\begin{array}{l}\text { Diversidade de } \\
\text { macrófitas }\end{array}$ \\
\hline Vegetação marginal & $\begin{array}{l}\text { Vegetação ciliar } \\
\text { escassa }\end{array}$ & $\begin{array}{l}\text { Vegetação ciliar } \\
\text { ausente }\end{array}$ & $\begin{array}{l}\text { Vegetação ciliar } \\
\text { ausente }\end{array}$ & $\begin{array}{l}\text { Vegetação ciliar } \\
\text { escassa }\end{array}$ & $\begin{array}{l}\text { Manchas de vegeta- } \\
\text { ção em recuperação }\end{array}$ \\
\hline Uso do solo & $\begin{array}{l}\text { Agricultura (trigo, soja } \\
\text { e milho) }\end{array}$ & $\begin{array}{l}\text { Agricultura (trigo, soja } \\
\text { e milho) e pecuária }\end{array}$ & $\begin{array}{l}\text { Agricultura (trigo, soja } \\
\text { e milho) e pecuária }\end{array}$ & $\begin{array}{l}\text { Agricultura (trigo, so- } \\
\text { ja e milho) e pecuária }\end{array}$ & $\begin{array}{l}\text { Agricultura (trigo, so- } \\
\text { ja e milho) e pecuária }\end{array}$ \\
\hline
\end{tabular}




\section{RESULTADOS E DISCUSSÃO}

Comparando-se a composição da dieta de $P$. squamosissimus no trecho Sertanópolis do rio Tibagi, ao longo do tempo foi verificado que em todos os períodos (1992/1993, 1994/1995 e 2001/2002) houve uniformidade na composição dos recursos alimentares consumidos, que foram agrupados em seis categorias: peixes, camarão, Odonata, Ephemeroptera, outros grupos de insetos e diversos (constituída de restos de vegetais, detritos e organismos que foram raramente encontrados) (Fig. 2).

Nesse trecho, a maior variação ocorreu no consumo de peixes, que diminuiu do primeiro para o último período. $O$ recurso que substituiu os itens da categoria peixes foi camarão, que aumentou em número, atingindo os maiores valores em composição percentual no último período. $\mathrm{O}$ mesmo ocorreu no rio Paranapanema nos trechos Cinzas, Cruzália e Porecatu, durante o mesmo período.

A comparação da composição percentual, pela análise de similaridade de Bray-Curtis, separou as amostras em dois grupos principais, um com maior composição percentual de peixes consumidos e outro com predomínio do item camarão (Fig. 3). O primeiro foi composto pelo trecho Sertanópolis nos períodos 1992/ 1993 e 1994/1995 e o trecho Primeiro de Maio. O segundo grupo foi composto pelos demais trechos, todos no período 2001/2002, mas ainda divididos em dois subgrupos: um formado pelos trechos Sertanópolis e Porecatu, onde o item camarão representou a maior composição percentual e o outro, pelos trechos Cinzas e Cruzália, como uma situação intermediária, onde o item camarão foi consumido em quantidades iguais ou levemente superiores às de peixes. Isto pode estar evidenciando que os alimentos principais da corvina são os peixes, mas com a redução na sua disponibilidade ela se torna oportunista, substituindo-os por outro alimento que esteja abundante.

Em todos os trechos dos rios Tibagi e Paranapanema e, em todas as épocas analisadas, a espécie se manteve como carnívora, mas nem sempre piscívora, pois o item camarão (Macrobrachium amazonicum) teve participação destacada na dieta de $P$. squamosissimus. BiAletzKi et al. (1997) relataram que esta espécie de crustáceo foi introduzida na bacia do rio Paraná junto com várias espécies de peixes, e que está estabelecida na planície inundável na região de Porto Rico. Nos trechos de influência da represa Capivara, da mesma forma que $P$. squamosissimus, pode-se considerar $M$. amazonicum estabelecida, e o trecho Tibagi (Sertanópolis) se destaca por ser o local com o maior percentual de camarão registrado na composição da dieta da corvina. Este trecho, que foi estudado em sua área marginal, é caracterizado por ter pouca profundidade, abundante vegetação aquática e em maior extensão que os demais trechos amostrados, podendo ser um ambiente mais favorável para M. amazonicum. Montoya (2003) também verificou que duas espécies de camarão do gênero Macrobrachium são encontradas associadas às raízes da vegetação aquática no delta do rio Orenoco e que, provavelmente, utilizam as raízes de macrófitas para se reproduzir.

Em reservatórios e outros ambientes da bacia do rio Paraná foram realizados vários estudos em que consta a análise da dieta da corvina. HAHN et al. (1998) analisando a dieta das espécies de peixes nos primeiros anos após a formação do reservatório Itaipu, verificaram que a corvina consumiu principalmente peixes e insetos. AlmEIDA et al. (1997) registraram o item camarão como comum na dieta da corvina coletada em trechos da planície de inundação do alto rio Paraná, mas mesmo assim, a espécie foi considerada como piscívora. Numa ampla investigação desta espécie durante os anos 1983 a 1988, no reservatório de Itaipu, HAHN et al. (1997), confirmaram a preferência por peixes, embora camarão tenha sido registrado na sua dieta. Vários fatores devem estar influenciando para que neste reservatório a corvina esteja se comportando de maneira distinta em relação aos trechos aqui analisados. Um destes fatores pode ser o maior número de espécies de peixes-presa disponíveis, pois aqueles autores registraram 37 espécies de peixes utilizadas pela corvina (contra 17 ou menos espécies por período, nesse estudo). Entretanto, a ausência de

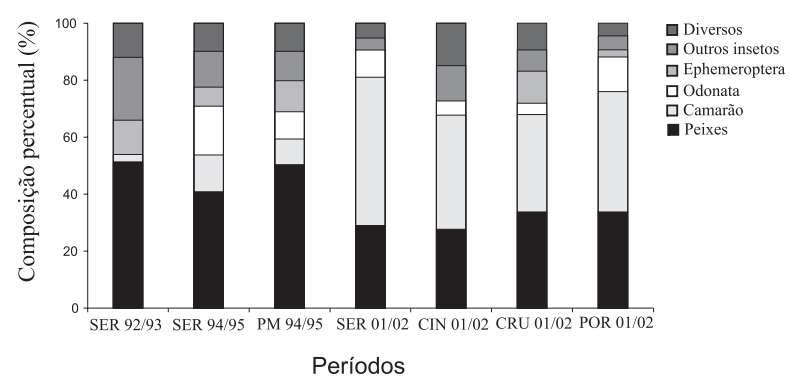

Fig. 2. Composição percentual dos recursos alimentares consumidos por $P$. squamosissimus nos trechos analisados nos rios Paranapanema (trechos CIN, Cinzas; CRU, Cruzália; POR, Porecatu) e Tibagi (trechos PM, Primeiro de Maio; SER, Sertanópolis) nos períodos 1992/1993, 1994/1995 e 2001/2002.

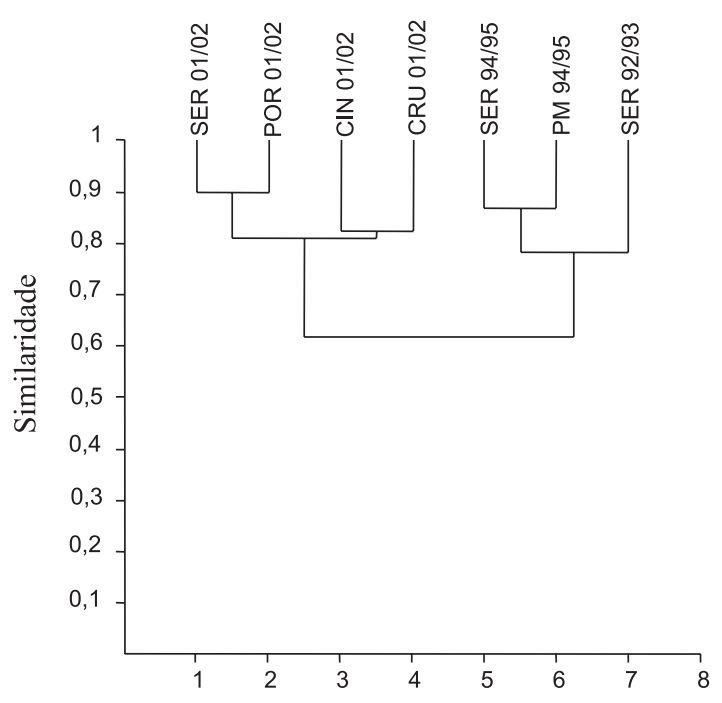

Fig. 3. Dendrograma de similaridade (coeficiente de Bray-Curtis), considerando a composição percentual dos recursos alimentares de Plagioscion squamosissimus nos trechos analisados nos rios Paranapanema (trechos CIN, Cinzas; CRU, Cruzália; POR, Porecatu) e Tibagi (trechos PM, Primeiro de Maio; SER, Sertanópolis) nos períodos 1992/1993, 1994/1995 e 2001/2002. 
Tabela II. Características das amostras de Plagioscion squamosissimus obtidas por análise de conteúdos estomacais por trecho e período de coleta (CIN, Cinzas; CRU, Cruzália; POR, Porecatu ; PM, Primeiro de Maio; SER, Sertanópolis).

\begin{tabular}{|c|c|c|c|c|c|c|c|}
\hline \multirow[t]{3}{*}{ Características das amostras } & \multicolumn{7}{|c|}{ Trechos amostrados } \\
\hline & \multicolumn{4}{|c|}{ Rio Tibagi } & \multicolumn{3}{|c|}{ Rio Paranapanema } \\
\hline & & SER & & $\mathrm{PM}$ & CIN & $\mathrm{CRU}$ & POR \\
\hline Período & $1992 / 1993$ & $1994 / 1995$ & $2001 / 2002$ & $1994 / 1995$ & $2001 / 2002$ & $2001 / 2002$ & $2001 / 2002$ \\
\hline Média de tipos de itens por indivíduo & 1,7 & 1,65 & 1,4 & 1,46 & 1,7 & 1,26 & 1,65 \\
\hline Total de exemplares analisados & 98 & 323 & 231 & 162 & 47 & 72 & 60 \\
\hline
\end{tabular}

acompanhamento da alimentação da corvina a partir daquela época não permite esclarecer se aquela população também alterou a sua alimentação, mostrando predomínio do camarão na dieta, como ocorreu nos trechos de influência da represa Capivara, onde o aumento e substituição de peixes por camarão na alimentação da corvina foram eventos recentes.

Nos trechos de influência da represa Capivara, além de encontrar um ambiente com características mais favoráveis nos trechos dos rios Tibagi e Paranapanema, o camarão pode estar sendo mais consumido pela corvina após a redução da abundância das espécies de peixespresa. Este fato pode ser evidenciado no estudo de um dos trechos aqui analisados e que foram discutidos por BenNEMANN \& ShiBATTA (2002). Estes autores constataram diminuição do número de indivíduos de algumas espéciespresa, principalmente Astyanax altiparanae, que foi consumida com maior freqüência pela corvina no período de 1992/1993.

Foram analisados 993 exemplares de $P$. squamosissimus, com maior número de exemplares para o rio Tibagi ( $82 \%$ do total; Tab. II). No trecho do rio Tibagi, em que foi possível acompanhar o comportamento da espécie ao longo do tempo, foi constatado que a atividade alimentar foi intensificada, pois houve um aumento crescente no número de exemplares com conteúdo estomacal. Nos trechos do rio Paranapanema, que foram investigados mais recentemente, os valores percentuais de indivíduos com alimento em seus estômagos foram os maiores encontrados dentre todos os trechos (Fig. 4). Junto com o fato da corvina mudar de alimento, substituindo peixes por camarão, houve mudança do comportamento quanto à freqüência na tomada de alimento, com grande aproveitamento na utilização de um recurso abundante no ambiente. Isto pode estar relacionado com a baixa seletividade de captura de presas pela corvina, que realiza uma varredura na coluna da água abrindo a boca e capturando o que encontra em sua frente (GouldiNG, 1980), facilitando a ingestão do camarão.

HAHN et al. (1999) avaliaram a atividade alimentar de $P$. squamosissimus no reservatório Itaipu e na planície de inundação do alto rio Paraná por vários períodos e verificaram baixos valores de freqüência de estômagos com alimento. Os autores associam a alta incidência de estômagos vazios ao hábito piscívoro da espécie. Da mesma forma, em sua bacia de origem, em lagos e na floresta inundada do rio Machado, Goulding (1980) verificou um grande número de exemplares com estômagos vazios. O mesmo foi observado no estudo do rio Paraná, e no rio Tibagi no período 1992/1993, quando

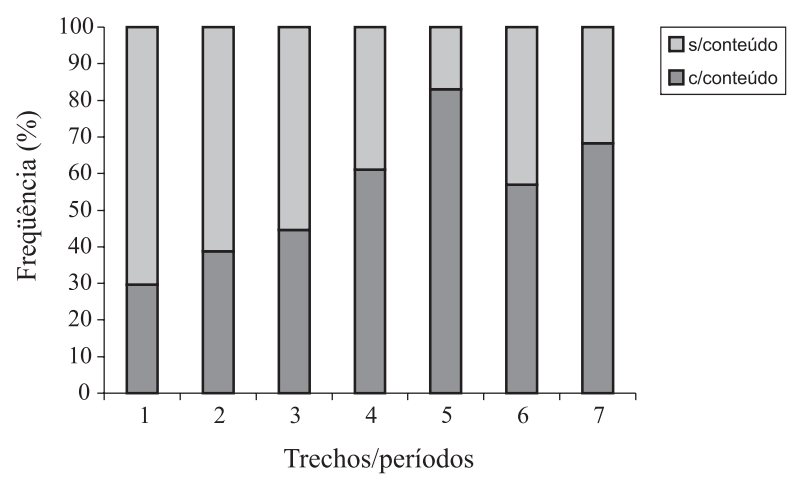

Fig. 4. Percentuais totais do número de exemplares com e sem conteúdo nos estômagos (1, rio Tibagi, Sertanópolis 1992/1993; 2, rio Tibagi, Sertanópolis 1994/1995; 3, rio Tibagi, Sertanópolis 2001/2002; 4, rio Tibagi, Primeiro de Maio 1994/1995; 5, rio Paranapanema, Cinzas 2001/2002; 6, rio Paranapanema, Cruzália 2001/2002; 7, rio Paranapanema, Porecatu 2001/2002).

a espécie consumiu preferencialmente peixes, como citado anteriormente.

Em outros rios e tipos de ambientes, também na região amazônica, GoULDING \& FERREIRA (1984) relataram que em alguns habitats, $P$. squamosissimus consome essencialmente camarão. Embora estes autores não façam referência sobre a relação da maior quantidade de indivíduos ingerindo camarão com o maior número de exemplares com conteúdo estomacal, foi registrado um número bem maior $(73,9 \%)$ do que havia sido citado por Goulding (1980), quando foi observado que poucos exemplares continham alimento no estômago na época e no habitat em que a espécie consumiu essencialmente peixes.

Os valores médios da riqueza de itens alimentares por indivíduo foram iguais ou menores que 1,7 itens alimentares em todas as épocas e em todos os trechos (Tab. II), mostrando que a maioria dos exemplares consome apenas um a dois tipos de itens por vez. O grande consumo de camarão contribuiu para a redução dos tipos de itens ingeridos por indivíduo, pois os menores valores médios foram verificados nos períodos mais recentes, no rio Tibagi (1,4 tipos de itens por indivíduo) em 2001/2002, quando a corvina utilizou o camarão em maior quantidade na sua dieta (Tab. II).

$\mathrm{Na}$ área de influência da represa Capivara, a composição da dieta das outras espécies de peixes mais abundantes, amostrados nos mesmos quatro trechos deste estudo, está sendo analisada. Nestas avaliações 
tem sido verificada a importância do item camarão na dieta de várias espécies de peixes. Ainda, o recurso camarão por ser abundante e ter encontrado um ambiente propício para seu desenvolvimento no trecho Sertanópolis do rio Tibagi, pode ser um dos fatores que manterá a abundância de P. squamosissimus nesta localidade, mesmo com o declínio das populações de suas espécies de peixes-presa.

Agradecimentos. Aos convênios UEL/COPATI/KLABIN e FAUEL/UEL/DUKE ENERGY pelo apoio e financiamento. Ao biólogo Mario Luis Orsi e aos técnicos do Museu de Zoologia da Universidade Estadual de Londrina, Edson Santana e Aparecido de Souza, pelas amostragens dos peixes. Ao Dr. Reinaldo Castro, pelas discussões e sugestões. Aos estagiários do curso de Ciências Biológicas, Wagner Galves Jr. e Murilo S. Dias, pela colaboração nas análises dos conteúdos estomacais.

\section{REFERÊNCIAS BIBLIOGRÁFICAS}

Almeida, V. L. L.; Hahn, N. S. \& Vazzoler, A. E. A. M. 1997. Feeding patterns in five predatory fishes of the high Paraná river floodplain (PR, Brazil). Ecology of Freshwater Fish 6:123-133.

Bennemann, S. T.; Shibatta, O. A. \& Garavello, J. C. 2000. Peixes do rio Tibagi: uma abordagem ecológica. Londrina, EDUEL. 62p.

Bennemann, S. T. \& Shibatta, O. A. 2002. Dinâmica de uma assembléia de peixes do rio Tibagi. In: Medri, M. E.; Bianchini, E.; Shibatta, O. A. \& Pimenta, J. A. eds. A bacia do rio Tibagi. Londrina, M. E. Medri. p.433-442.

Bialetzki, A.; Nakatani, K.; Baumgartner, G. \& Bond-Buckup, G. 1997. Occurrence of Macrobrachium amazonicum (Heller) (Decapoda, Palaemonidae) in Leopoldo's inlet (Ressaco do
Leopoldo), upper Paraná river, Porto Rico, Paraná, Brazil. Revista Brasileira de Zoologia 14(2):379-390.

Goulding, M. 1980. The fishes and the forest. Explorations in Amazonian Natural History. Berkeley, University of California Press. 280p.

Goulding, M. \& Ferreira, E. 1984. Shrimp-eating fishes and a case of prey switching in Amazon rivers. Revista Brasileira de Zoologia 2(3):85-97.

Hahn, N. S.; Agostinho, A. A. \& Goitein, R. 1997. Feeding ecology of curvina Plagioscion squamosissimus (Heckel, 1840) (Osteichthyes, Perciformes) in the Itaipu reservoir and Porto Rico floodplain. Acta Limnologica Brasiliensia 9:11-22.

Hahn, N. S.; Agostinho, A. A.; Gomes, L. C. \& Bini, L. M. 1998 Estrutura trófica da ictiofauna do reservatório de Itaipu (Paraná-Brasil) nos primeiros anos de formação. Interciencia 23(5):299-305.

Hahn, N. S.; Loureiro, V. E. \& Delariva, R. L. 1999. Atividade alimentar da curvina Plagioscion squamosissimus (Heckel, 1840) (Perciformes, Scianidae) no rio Paraná. Acta Scientiarum 21(2):309-314.

Hammer, O.; Harper, D. A. T. \& Ryan, P. D. 2003. PAST Palaeontological Statistics, ver. 1.12. Disponível em: $<$ http://www.folk.uio.no/ohammer/past $>$. Acesso em: 31.07 .2003$.

Hynes, H. B. N. 1950. The food of fresh-water sticklebacks (Gasterosteus aculeatus and Pygosteus pungitius), with a review of methods used in studies of the food of fishes. Journal of Animal Ecology 19:36-57.

Lowe-McConnell, R. 1999. Estudos ecológicos de comunidades de peixes tropicais. São Paulo, EDUSP, $534 \mathrm{p}$.

Montoya, J. V. 2003. Freshwater shrimps of the genus Macrobrachium associated with roots of Eichhornia crassipes (water hyacinth) in the Orinoco delta (Venezuela). Caribbean Journal of Science 29(1):155-159. 